\title{
Postnatal diagnosis of harlequin ichthyosis a case report
}

\begin{abstract}
Objective: Ichthyoses are cornification disorders in which irregular epidermal separation and desquamation result in a faulty epidermal membrane. Harlequin ichthyosis (HI) was a rare and extreme type that led to neonatal death. It was caused by mutations in the ABCA12 gene, and the inheritance pattern is autosomal recessive.
\end{abstract}

Case report: We present a case of HI that was diagnosed postnatally by clinical review. Extreme ectropion, eclabium, flattened nose, and primitive ears were discovered in the fetus. As a result of HI complications, the fetus died.

Conclusion: The presence of HI was linked to a poor prognosis and a high mortality rate. Prenatal ultrasound and genetic analysis were critical for prenatal diagnosis of HI, but genetic modalities were not available and were prohibitively costly, despite their utility in providing appropriate prenatal therapy to families with HI babies. This case was recorded because of its rarity, as well as to draw attention to the connection between.

Keywords: harlequin ichthyosis, ABCA12, atbara, River Nile state, Sudan
Volume 7 Issue 2 - 202I

\author{
Mohammed Ahmed Ibrahim Ahmed,' \\ Mohamed Ali Saad Mohamed,' Salwa Ahmed \\ Mohammed Abbas,' Athar Asim Ahmed \\ Mohammed,' Nosiba Ibrahim Hammed \\ Alyamani ${ }^{2}$ \\ 'Department of Obstetrics \& Gynecology, Atbara Teaching \\ Hospital, Sudan \\ ${ }^{2}$ Department of Pediatric, NICU,Atbara Teaching Hospital, \\ Sudan
}

\author{
Correspondence: Mohammed Ahmed Ibrahim \\ Ahmed, Assistant professor of Microbiology, Nile Valley \\ University, Faculty of Medicine, House officer Obstetrics \\ \& Gynecology,Atbara Teaching Hospital,Atbara, Sudan, \\ Tel +2490/22570655/+2499/2656095, \\ Emailmohammedabukleewa@gmail.com, \\ Abukleewa1974@yahoo.com
}

Received: March 29, 2021 | Published: April 19, 2021

Abbreviations: ABCA12, adenosine triphosphate binding cassette A12; HI, harlequin ichthyosis; NICU, neonatal intensive care unit

\section{Introduction}

Autosomal recessive congenital ichthyosis (ARCI) is a diverse group of disorders that occur at birth with generalized skin involvement and no other organ system manifestations. Lamellar ichthyosis is estimated to affect one in every 200,000 to 300,000 people. ${ }^{1} \mathrm{HI}$ is a very rare congenital condition with a high perinatal mortality rate. In most cases, neonates with $\mathrm{HI}$ do not make it through the first year of life. ${ }^{2}$ The phenotype continuum of classic lamellar ichthyosis and nonbullous congenital ichthyosiformerythroderma, self-healing collodion infant, acral self-healing collodion baby, bathing suit ichthyosis, and harlequin ichthyosis are all included in ARCI. The mode of inheritance is autosomal or X-linked. ${ }^{3}$ To date, mutations in at least six genes have been identified. ${ }^{4}$ In suspicious cases, fetal skin biopsy electron microscopy and DNA-based diagnosis with chorionic villus sampling or amniocentesis can be used to validate the antenatal diagnosis. ${ }^{5}$

\section{Case report}

A 29-year-old woman with drainage of liquor and supra pubic pain was seen in the emergency department of the obstetrical and gynecology Atbara teaching hospital. Her previous vaginal deliveries have all been uneventful, with stable and alive babies; the most recent delivery resulted in a male at the age of four. The baby was the third child born to a consanguineous couple, who were maternal first cousins. Preterm labour, frequent miscarriages/stillbirths, genetic abnormalities in older children, or hereditary dermatological conditions were not reported by the parents. That was an event that had not been pre-booked. During her first and second trimesters, she had an antenatal care record that showed no abnormalities. The height of the fundus was approximately equal to about 34 weeks of gestation, and the fetal head was engaged, according to an abdominal examination.

For prevention of intra-partum infection and fetal lung immaturity, she got one shot of intravenous ceftriaxone $1 \mathrm{gm}$, metronidazole, and erythromycin tabs $250 \mathrm{mg}$ orally/6 nightly, and intramuscular dexamethasone. The labor passed at its own pace.

A female infant of weight $1.7 \mathrm{~kg}$ was conveyed. At the time of birth, the body of the neonate was secured with resembling cloak shield, dense and widespread pale brownish scales; Erythematous fissures ran deep into the dermis between these keratin layers of scales. On the body, the scales and fissures formed a diamond grid, which was highlighted in the chest. Per arm and digit was rigidly semi-flexed. Other obvious characteristics included a mouth that is both set and fully open, slanted nasal bridge, primitive external ears, and ectropion and eclabium. Although there were no brows or eyelashes, scalp hair was visible. There were no natural orifices that were not patentable. The pediatrician and dermatologist were advised, and a clinical diagnosis of Harlequin Ichthyosis Congenita was made, marking the first time such a diagnosis had been made at Atbara Teaching Hospital (Figure 1).

The neonate was admitted to the neonatal intensive care unit(NICU) after the family underwent counseling, to avoid any instances of aspiration, she was begun on parenteral feeding and intravenous antibiotics via an umbilical line. Regular saline was used to disinfect the skin, every two to four hours, liquid paraffin and emollient were softly added to the entire cutaneous surface. In addition, a topical antibiotic (Fuciderm cream) was applied to the skincare regimen, Optiflox, an antibacterial eye drop, was used (Figure 2). 




Figure I A neonate with $\mathrm{HI}$ at labor rom.

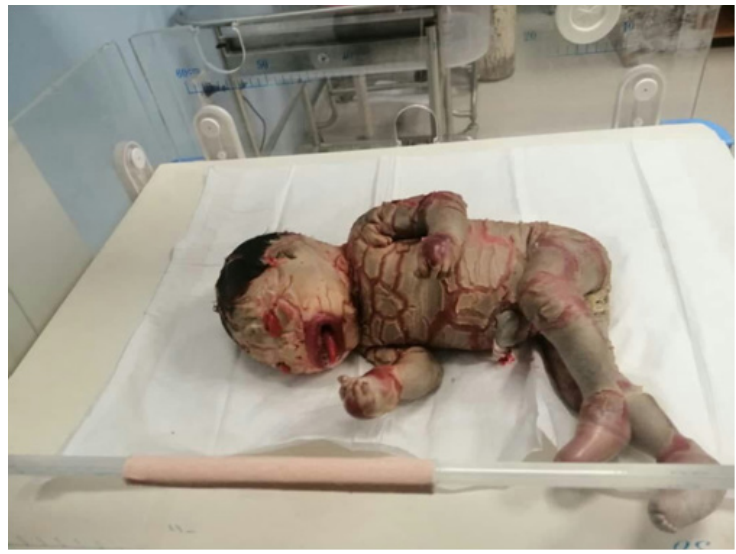

Figure 2 A neonate with $\mathrm{HI}$ at $\mathrm{NICU}$.

On day four, oral feeding was initiated, but there was a weak response. When she is left on one side for an extended period of time smells awful. She was experienced respiratory problems within six days of birth, despite the compassionate management, and became tachypnic and bradycardic by day five, not responding well to oxygen because her left nasal nostril was covered. The newborn died on the sixth day of life as a result of respiratory distress. The parents were granted psychological assistance, they were told of the disease's implications, and genetic evaluation was prescribed for potential births.

\section{Discussion}

$\mathrm{HI}$ is a rare autosomal recessive disease with a high rate of recurrence. ABCA12 DNA monitoring may be given to suspicious cases and families that have already been affected. Facial disfigurement, a broad open mouth, and the disappearance of normal nasal features are prenatal ultrasonography findings reminiscent of a harlequin fetus. a wide open mouth, no standard nasal morphology, in front of the eyes showed split cystic formations, the lack of normal ear morphology, a rugged exterior, rigid limbs in a semi-flexed posture, limited fetal movement, limb abnormalities characterized by hypoplastic fingertips, toes, as well as short phalanges, clubfoot, contracted fingers, high echogenicity amniotic fluid, there are no related visceral abnormalities.

Explaining 2D images and diagnosing $\mathrm{HI}$ are all made possible by $3 \mathrm{D}$ imaging. The distinctive characteristics of prenatal ultrasound imaging typically occur later in the pregnancy. As a result, even though the second trimester anatomy scan is regular, scans should be replicated. Furthermore, where a DNA diagnosis is inaccessible, these scans may be helpful. ${ }^{6}$ The significance of perinatal diagnosis can't be overstated. An ultrasound and an analysis of amniotic fluid cells, specifically, of the fetal mouth, especially in the 17th week of gestation, have shown to produce significant results. Also included is a detailed history of the family's backgrounds, as well as consanguinity between the kin, details concerning prior pregnancies, as well as whether or not other children have a genodermatosis since birth are relevant. A skin biopsy will likely show anatomical defects of lamellar granules and epidermal keratin expression in the postnatal diagnosis. The grotesque presentation of the fetus is usually enough to make a diagnosis.?

In subsequent births, the rate of recurrence was more than 20 percent. HI was instantly diagnosed at birth due to its extreme clinical characteristics. However, with the advancement in prenatal testing procedures, few cases of HI with a family background had been diagnosed prenatally, and the parents had undergone adequate counseling leading up to the births of the babies. ${ }^{8}$

HI, like several other chronic skin disorders, necessitate careful management and outpatient check-up, in order to achieve long-term sustainability. Community social support, including certain patient support groups, play a significant role in supporting the patient's and family's psycho-social quality of life, which is unrealistic in our condition. Individuals with chronic, debilitating skin disorders can face social and cultural discrimination, which can exacerbate clinical outcomes, especially in areas where traditional health and disease beliefs remain

In the absence of a family history of genodermatosis, we reported this unusual case of HI. Nevertheless, a prenatal standardized 3D scan, a diagnostic genetic work-up, and a hyper keratotic histological portion of a skin biopsy disclosed that the baby had HI.

\section{Conclusion}

Harlequin icthyosis is a deadly geno dermatological presentaton. In families with consanguineous marriages, parental counseling is of significant value, along with genetic diagnosis for a mutant adenosine triphosphate binding-cassette transporter ABCA12 gene in high-risk individual. Icthyosis type, sophisticated NICU facilities, availability of a multidisciplinary group with advanced qualifications, and dependable approaches for early detection of $\mathrm{HI}$ can reduce tension for family members and health care providers, resulting in a more satisfying outcome. The medical and social effects of congenital HI can be catastrophic. As a result, the family should obtain sufficient social and psychological assistance.

\section{Prognosis}

The most frequent cause of death in such clinical presentation is fulminant neonatal sepsis. Attempting to control temperature, intended to prevent dehydration and infections, managing skin conditions, and delivering physical therapy can all help to improve management outcomes.

\section{Acknowledgments}

For their support and verbal informed consent for the publication of this case study, we thank Baby's parents.

\section{Conflicts of interest}

The author claim that the publishing of this case report would not entail any conflicts of interest. 


\section{Funding}

None.

\section{References}

1. Yang JM, Ahn KS, Cho MO, et al. Novel mutations of the transglutaminase 1 gene in lamellar ichthyosis. Journal of Investigative Dermatology. $2001 ; 117(2): 214-218$.

2. Shibata A, Akiyama M. Epidemiology, medical genetics, diagnosis and treatment of harlequin ichthyosis in Japan. Pediatr Int. 2015;57:516-522.

3. Vinzenz Oji, Gianluca Tadini, Masashi Akiyama, et al. Revised nomenclature and classification of inherited ichthyoses:results of the first Ichthyosis Consensus Conference in Sorze 2009. Journal of the American Academy of Dermatology. 2010;63(4):607-641.
4. Fischer J. Autosomal recessive congenital ichthyosis. Journal of Investigative Dermatology. 2009;129(6):1319-1321.

5. Jilumudi UB. Harlequin Ichthyosis: A medico legal case report \& review of literature with peculiar findings in autopsy. J Forensic Leg Me. 2012;19:352-354

6. Vijayakumari M, Reddy KD, Routhu M, et al. Prenatal diagnosis of harlequin ichthyosis: a case report. Obstet Gynecol Sci. 2020;63(1):94-97.

7. Tahir A, Tariq S, Haider S Ali, et al. Ichthyosis Congenita, Harlequin Type: A Fatal Case Report. Cureus. 2018;10(10):e3524.

8. Wei Jian, Qi-Ting Du, Zhen-Fei Lai, et al. Case Report. Prenatal diagnose of a fetus with Harlequin ichthyosis in a Chinese Family. Taiwanese Journal of Obstetrics \& Gynecology. 2018;57:452e455. 